\title{
Correction: OpinionML-Opinion Markup Language for Sentiment Representation. Symmetry 2019, 11, 545
}

\author{
Mohammed Attik ${ }^{1}\left(\mathbb{D}\right.$, Malik Muhammad Saad Missen ${ }^{2}\left({ }^{\circ}\right.$, Mickaël Coustaty ${ }^{3}{ }^{\circledR}$, \\ Gyu Sang Choi ${ }^{4, *}{ }^{(0)}$, Fahd Saleh Alotaibi ${ }^{5}\left(\mathbb{D}\right.$, Nadeem Akhtar ${ }^{2}{ }^{(0}$, Muhammad Zeeshan Jhandir ${ }^{2}$, \\ V. B. Surya Prasath ${ }^{6}{ }^{\circ}$, Nadeem Salamat ${ }^{7(-)}$ and Mujtaba Husnain ${ }^{2}{ }^{(1)}$ \\ 1 Opinaka Lab, 97 rue Freyr, 34000 Montpellier, France; m.attik@opinaka.com \\ 2 Department of Computer Science \& IT, The Islamia University, Bahawalpur 63100, Pakistan; \\ saad.missen@iub.edu.pk (M.M.S.M.); nadeem.akhtar@iub.edu.pk (N.A.); \\ zeeshan.jhandir@iub.edu.pk (M.Z.J.); mujtaba.husnain@iub.edu.pk (M.H.) \\ 3 L3i Lab, Université of La Rochelle Av. Michel Cŕepeau, 17000 La Rochelle, France; \\ mickael.coustaty@univ-lr.fr \\ 4 Department of Information and Communication Engineering, Yeungnam University, \\ Gyeongsan 712-749, Korea \\ 5 Faculty of Computing and Information Technology, King Abdulaziz University, Jeddah 21577, Saudi Arabia; \\ fsalotaibi@kau.edu.sa \\ 6 Department of Pediatrics Cincinnati, Children's Hospital Medical Center, Cincinnati, OH 45229 USA; \\ prasatsa@uc.edu \\ 7 Department of Mathematics, Khawaja Fareed University of Engineering and Technology, \\ Rahim Yar Khan 64200, Pakistan; nadeem.salamat@kfueit.edu.pk \\ * Correspondence: castchoi@ynu.ac.kr
}

Received: 16 January 2020; Accepted: 17 January 2020; Published: 22 January 2020

The authors wish to make the following corrections to their paper [1]:

The authorship should be changed from

Malik Muhammad Saad Missen ${ }^{1}$, Mickaël Coustaty ${ }^{2}$, Gyu Sang Choi ${ }^{3, *}$, Fahd Saleh Alotaibi ${ }^{4}$, Nadeem Akhtar ${ }^{1}$, Muhammad Zeeshan Jhandir ${ }^{1}$, V. B. Surya Prasath ${ }^{5}$, Nadeem Salamat ${ }^{6}$ and Mujtaba Husnain ${ }^{1}$

1 Department of Computer Science \& IT, The Islamia University, Bahawalpur 63100, Pakistan

2 L3i Lab, Université of La Rochelle Av. Michel Cŕepeau, 17000 La Rochelle, France

3 Department of Information and Communication Engineering, Yeungnam University, Gyeongsan 712-749, Korea

4 Faculty of Computing and Information Technology, King Abdulaziz University, Jeddah 21577, Saudi Arabia

5 Department of Pediatrics Cincinnati, Children's Hospital Medical Center, Cincinnati, $\mathrm{OH} 45229$ USA

6 Department of Mathematics, Khawaja Fareed University of Engineering and Technology, Rahim Yar Khan 64200, Pakistan

to the following correct version:

Mohammed Attik ${ }^{1}$, Malik Muhammad Saad Missen ${ }^{2}$, Mickaël Coustaty ${ }^{3}$, Gyu Sang Choi ${ }^{4, *}$, Fahd Saleh Alotaibi ${ }^{5}$, Nadeem Akhtar ${ }^{2}$, Muhammad Zeeshan Jhandir ${ }^{2}$, V. B. Surya Prasath ${ }^{6}$, Nadeem Salamat ${ }^{7}$ and Mujtaba Husnain ${ }^{2}$

1 Opinaka Lab, 97 rue Freyr, 34000 Montpellier, France; m.attik@opinaka.com 
2 Department of Computer Science \& IT, The Islamia University, Bahawalpur 63100, Pakistan; saad.missen@iub.edu.pk (M.M.S.M.); nadeem.akhtar@iub.edu.pk (N.A.); zeeshan.jhandir@iub.edu.pk (M.Z.J.); mujtaba.husnain@iub.edu.pk (M.H.)

3 L3i Lab, Université of La Rochelle Av. Michel Cŕepeau, 17000 La Rochelle, France; mickael.coustaty@univ-lr.fr

4 Department of Information and Communication Engineering, Yeungnam University, Gyeongsan 712-749, Korea

5 Faculty of Computing and Information Technology, King Abdulaziz University, Jeddah 21577, Saudi Arabia; fsalotaibi@kau.edu.sa

6 Department of Pediatrics Cincinnati, Children's Hospital Medical Center, Cincinnati, OH 45229 USA; prasatsa@uc.edu

7 Department of Mathematics, Khawaja Fareed University of Engineering and Technology, Rahim Yar Khan 64200, Pakistan; nadeem.salamat@kfueit.edu.pk

The authors would like to apologize for any inconvenience caused to the readers by these changes. The changes do not affect the scientific results. The manuscript will be updated and the original will remain online on the article webpage, with a reference to this Correction.

\section{Reference}

1. Missen, M.M.S.; Coustaty, M.; Choi, G.S.; Alotaibi, F.S.; Akhtar, N.; Jhandir, M.Z.; Prasath, V.B.S.; Salamat, N.; Husnain, M. OpinionML-Opinion Markup Language for Sentiment Representation. Symmetry 2019, 11, 545.

(C) 2020 by the authors. Licensee MDPI, Basel, Switzerland. This article is an open access article distributed under the terms and conditions of the Creative Commons Attribution (CC BY) license (http://creativecommons.org/licenses/by/4.0/). 